\title{
Zum Tode von Professor Dr. iur. Dr. h.c. Adolf Laufs
}

Adolf Laufs ist am 3. Januar 2014 verstorben. Die deutsche Rechtswissenschaft verliert einen ihrer hervorragendsten Vertreter, das Medizinrecht den Doyen. Laufs hat das Arztund Medizinrecht als eigenständige Disziplin ausgeformt, dessen Entwicklung über fast ein halbes Jahrhundert nachhaltig beeinflusst und wesentlich geprägt.

Am 18. November 1935 als Sohn einer kinderreichen Arztfamilie in Tuttlingen geboren und dort aufgewachsen, studierte Laufs ab 1955 Rechtswissenschaft an der AlbertLudwigs-Universität Freiburg, zwischenzeitlich an der Freien Universität Berlin und während seines Referendariats an der Hochschule für Verwaltungswissenschaften in Speyer. 1961 promovierte er bei Hans Thieme in Freiburg, nach dem Assessorexamen 1963 folgte eine kurze anwaltliche Tätigkeit in seiner Heimatstadt, 1968 habilitierte er sich an der Freiburger Juristenfakultät. Ein früher Ruf führte Laufs 1969 nach Heidelberg auf den Lehrstuhl für Deutsche Rechtsgeschichte, Deutsches Privatrecht und Bürgerliches Recht an der Ruprecht-Karls-Universität, wo er als Direktor dem Institut für geschichtliche Rechtswissenschaft vorstand. Hier blieb er, als ihn Rufe an andere Universitäten ereilten. In den Jahren 1979 bis 1983 übernahm er das Amt des Rektors der Universität Heidelberg. 1984 folgte er dann doch einem Ruf an die Eberhard-Karls-Universität Tübingen, kehrte vier Jahre später aber wieder auf seinen Heidelberger rechtshistorischen Lehrstuhl zurück. Stets begleitete ihn seine Frau Christina, mit der er eine überaus glückliche Ehe führte, aus der fünf Kinder hervorgingen.

Das wissenschaftliche Werk von Adolf Laufs ist weit gespannt. Rechts- und Verfassungsgeschichte waren seine Wurzeln und Heimstätte, Deutsches Privatrecht und das Bürgerliche Recht sein Metier, Medizinrecht seine Leidenschaft und Berufung. Laufs hat Dutzende Monographien verfasst und herausgegeben, unzählige Aufsätze, Urteilsanmerkungen und Rezensionen geschrieben.

Dissertation und Habilitation galten rechtsgeschichtlichen Themen. 1973 erschien das viel beachtete Lehrbuch „Rechtsentwicklungen in Deutschland“ (6. Auflage 2006). Von 1978 bis 2000 war Laufs Mitherausgeber der SavignyZeitschrift für Rechtsgeschichte. 2005 initiierte er mit anderen die Gründung der „Heidelberger Rechtshistorischen Gesellschaft“.

Das Medizinrecht hat Laufs wie kein anderer durchdrungen, beherrscht und kunstvoll entfaltet. Seine Abhandlungen zu den Rechtsfragen menschlichen Lebens, dessen Anfang und dessen Ende, haben höchsten sittlichen Wert. Die Ausführungen des Zivilrechtlers zu Haftungsfragen gaben der Rechtspraxis viele wichtige Impulse. Seit Habilitationstagen beschäftigte Laufs das große Thema der Willensfreiheit im Recht, dem er immer wieder nachging (s. zuletzt MedR 2011, S. 1 ff.). Von den vielen Veröffentlichungen besonders erwähnt seien das 1977 erschienene „Arztrecht“ (6. Auflage 2009), das 1992 gemeinsam mit Wilhelm Uhlenbruck herausgegebene „Handbuch des Arztrechts" (4. Auflage 2010) und die regelmäßigen Berichte und Kommentare in der NJW. Von 1985 bis 2006 war Laufs Schriftleiter der ,MedR“, zweiundzwanzig Jahre lang hat er die Beiträge redigiert, insgesamt 264 Ausgaben zusammengestellt, erst gemeinsam mit Helmut Narr, ab 1991 gemeinsam mit Hans-Jürgen Rieger. Bis kurz vor seinem Tode hat er Abhandlungen für ,seine“ MedR verfasst.
Adolf Laufs hat viele Aufgaben in Gremien der Wissenschaft und des Staates übernommen. Als Rektor führte er seine Universität aus einer immer noch unruhigen Zeit und er schloss sechs Partnerschaften der „Ruperto Carola“, darunter mit der Universität Montpellier und mit der Hebräischen Universität Jerusalem. Er wirkte an der Gründung dreier Juristischer Fakultäten mit, der Universitäten Trier und Konstanz in den siebziger Jahren, nach der Wiedervereinigung der Universität Dresden. Seit 1976 gehörte Laufs der Heidelberger Akademie der Wissenschaften an. Er war langjähriger Vorsitzender der Akademiekommission für das Deutsche Rechtswörterbuch. Über Jahrzehnte engagierte er sich als Mitglied der Ethikkommission bei der Landesärztekammer Stuttgart, in den Ethikkommissionen der Universitäten Heidelberg und Tübingen, bis 2002 zudem als Mitglied der Zentralen Kommission zur Wahrung ethischer Grundsätze in der Medizin und ihren Grenzgebieten. Laufs war Gründungsmitglied der Deutschen Gesellschaft für Medizinrecht und von 1990 bis 1992 deren Präsident, seit 1998 Mitdirektor des Instituts der Universitäten Heidelberg und Mannheim für deutsches, europäisches und internationales Medizinrecht, Gesundheitsrecht und Bioethik, von 1978 bis 2000 Mitglied des Vorstandes der Kommission für geschichtliche Landeskunde in Baden-Württemberg. Für seine Verdienste um Universität, Staat und Gesellschaft wurde er vielfach ausgezeichnet, unter anderem mit der Ehrendoktorwürde der Universität Montpellier, dem Bundesverdienstkreuz des Verdienstordens der Bundesrepublik Deutschland, der Ehrenmitgliedschaft der Deutschen Gesellschaft für Rechtsmedizin, dem Ehrenzeichen der Deutschen Ärzteschaft.

Adolf Laufs war nicht nur ein großer Wissenschaftler, sondern auch ein faszinierender akademischer Lehrer, der Generationen von jungen Juristen prägte. Wenn er unterrichtete, waren die Hörsäle stets vollbesetzt, ob in Heidelberg, in Tübingen oder in Dresden während seiner hauptamtlichen Abordnung 1992/1993. Laufs vermochte in anspruchsvollen Veranstaltungen die schwierigsten Rechtsfragen verständlich darzustellen und die Hörer für die Materie zu begeistern. Seine Studenten behalten einen Hochschullehrer mit Leib und Seele in Erinnerung. Auch außerhalb der Veranstaltungen war Laufs immer für sie da, stand als Gutachter zur Verfügung, setzte sich als Prüfer ein und führte trotz hoher Ansprüche viele Schüler an das Ziel.

Stets verteidigte Laufs ,,die Idee der Universität“ gegen vermeintliche technokratische Notwendigkeiten. Er verfocht die Humboldtschen Ideale „Einsamkeit und Freiheit“. Eine Unterwerfung der Forschung unter reines Nutzenkalkül oder Profitinteresse der Wirtschaft hat er nie akzeptiert: Wissenschaft muss auf Wahrheitssuche abzielen, auf Erkenntnis um ihrer selbst willen.

Laufs tiefe Menschlichkeit, seine Aufgeschlossenheit und Bescheidenheit, Toleranz und Loyalität haben viele Freundschaften begründet. Die große Festschrift „Humaniora. Medizin - Recht - Geschichte“ zu seinem 70. Geburtstag ist Ausdruck der allseitig hohen Wertschätzung. Wir werden Adolf Laufs, den stets anregenden Austausch mit ihm, seine umfassende Bildung und seine reichen Erfahrungen auf allen Lebensgebieten, seine Empathie und seine menschliche Wärme sehr vermissen. 